\title{
Treatment completion for latent tuberculosis infection in Norway: a prospective cohort study
}

Yvette Louise Schein', Tesfaye Madebo ${ }^{2}$, Hilde Elise Andersen ${ }^{3}$, Trude Margrete Arnesen ${ }^{4}$, Anne Ma Dyrhol-Riise ${ }^{5,6,7}$, Hallgeir Tveiten ${ }^{8}$, Richard A. White ${ }^{9}$ and Brita Askeland Winje ${ }^{10^{*}}$ (D)

\begin{abstract}
Background: Successful treatment of latent tuberculosis infection (LTBI) is essential to reduce tuberculosis (TB) incidence rates in low-burden countries. This study measures treatment completion and determinants of noncompletion of LTBI treatment in Norway in 2016.

Methods: This prospective cohort study included all individuals notified with LTBI treatment to the Norwegian Surveillance System for Infectious Diseases (MSIS) in 2016. We obtained data from MSIS and from a standardized form that was sent to health care providers at the time of patient notification to MSIS. We determined completion rates. Pearson's chi squared test was used to study associations between pairs of categorical variables and separate crude and multivariable logistic regression models were used to identify factors associated with treatment completion and adverse drug effects.
\end{abstract}

Results: We obtained information on treatment completion from 719 of the 726 individuals notified for LTBI treatment in 2016. Overall, 91\% completed treatment. Treatment completion was highest in the foreign-born group [foreign-born, $n=562$ (92\%) vs Norwegian-born, $n=115(85 \%), p=0.007]$. Treatment completion did not differ significantly between prescribed regimens $(p=0.124)$. Adverse events were the most common reason for incomplete treatment. We found no significant differences in adverse events when comparing weekly rifapentine (3RPH) with three months daily isoniazid and rifampicin $(3 \mathrm{RH})$. However, there were significantly fewer adverse events with $3 \mathrm{RPH}$ compared to other regimens $(p=0.037)$. Age over 35 years was significantly associated with adverse events irrespective of regimen $(p=0.024)$, whereas immunosuppression was not significantly associated with adverse events after adjusting for other variables $(p=0.306)$. Treatment under direct observation had a significant effect on treatment completion for foreignborn (multivariate Wald $p$-value $=0.017$ ), but not for Norwegian-born (multivariate Wald $p$-value $=0.408$ ) individuals.

Conclusions: We report a very high treatment completion rate, especially among individuals from countries with high TB incidence. The follow-up from tuberculosis-coordinators and the frequent use of directly observed treatment probably contributes to this. Few severe adverse events were reported, even with increased age and in individuals that are more susceptible. While these results are promising, issues of cost-effectiveness and targeting treatment to individuals at highest risk of TB are important components of public health impact.

Keywords: Latent tuberculosis infection, Preventive treatment, Rifapentine, Isoniazid, Rifampicin, Chemoprophylaxis, Compliance, Screening, Surveillance

\footnotetext{
* Correspondence: brita.winje@fhi.no

${ }^{10}$ Department of Vaccine Preventable Diseases, Norwegian Institute of Public

Health, Oslo, Norway

Full list of author information is available at the end of the article
}

(c) The Author(s). 2018 Open Access This article is distributed under the terms of the Creative Commons Attribution 4.0 International License (http://creativecommons.org/licenses/by/4.0/), which permits unrestricted use, distribution, and reproduction in any medium, provided you give appropriate credit to the original author(s) and the source, provide a link to the Creative Commons license, and indicate if changes were made. The Creative Commons Public Domain Dedication waiver (http://creativecommons.org/publicdomain/zero/1.0/) applies to the data made available in this article, unless otherwise stated. 


\section{Background}

Treatment of latent tuberculosis infection (LTBI) in groups at high risk for tuberculosis (TB) disease is a cornerstone in the global strategy to eliminate TB [1]. Norway has a mandatory screening program for TB and LTBI, which includes immigrants from high TB incidence countries, pre-employment screening, and outbreak management [2]. In addition, LTBI management is recommended prior to iatrogenic immunosuppression. In 2016, immigrants under the age of 35 from countries with TB incidence rates (IR) $>40$ per 100,000 population (as estimated by WHO) were eligible for screening with IGRA (or equivalent) upon arrival in Norway. In addition, immigrants $\geq 15$ years of age were screened for TB with a chest X-ray. In Norway's national guidelines, LTBI treatment is strongly recommended for children under the age of 5, contacts, those with fibrotic lesions on chest X-ray, or those with select immunosuppressive conditions (HIV-infection, haemodialysis, solid organ transplants, malignancies, or prior to iatrogenic immunosuppression). LTBI treatment is conditionally recommended for children aged 5-14 years, those with calcifications on chest X-ray, those who are underweight, and individuals with long-term steroid treatment, diabetes mellitus, or drug addiction. Being foreign-born was not considered a single criterion for priority for treatment at the time of the study [3].

The use of LTBI treatment has rapidly increased in Norway in recent years, stabilizing at around 750 cases per year $[4,5]$. Individuals prescribed LTBI treatment are reported to the Norwegian Surveillance System for Infectious Diseases (MSIS) [6]. However, treatment completion, an important indicator of the impact, safety, and cost effectiveness of the screening program, is not routinely reported.

LTBI treatment completion rates vary considerably across risk-groups and settings [7], and long duration and adverse events are well-known barriers to treatment completion [7-12]. A Norwegian study from 2009 found overall high LTBI treatment completion, and no adverse events requiring hospital admission [13]. Since then, Norwegian guidelines have increasingly targeted groups at high risk for TB reactivation, including older individuals with more comorbidities and recent immigrants to Norway [3]. Recent immigrants are often difficult to follow-up due to their mobility and a lack of governmentissued identification numbers [14, 15]. Additionally, the increasing use of immunosuppressants may pose new challenges to LTBI treatment strategy.

The most common LTBI regimen is a daily combination of rifampicin and isoniazid for three months $(3 \mathrm{RH})$. The increasing use of weekly-administered rifapentine (3RPH) [8], a newer and less-studied regimen, highlights the need for strengthened LTBI treatment surveillance. National guidelines currently recommend directly observed treatment (DOT) for this new 3RPH regimen.

Severe adverse events due to preventive treatment are primarily related to isoniazid induced peripheral neuropathy and hepatotoxicity, with higher rates found in those aged $\geq 35$ years $[12,16]$, and hepatotoxicity, gastrointestinal intolerance, and hypersensitivity from rifamycins [12]. We question whether tolerance to adverse effects may be context specific, with lower tolerance in a country where TB is rare. If significant scale-up of LTBI treatment is indicated, information about adverse events and treatment completion by regimen will be highly relevant.

In Norway, prescription of TB drugs, including LTBI treatment, is the responsibility of pulmonologists, infectious disease specialists, or paediatricians who work primarily in hospital settings. The role of "TB coordinator" was introduced in the national TB control programme in 2003 to strengthen patient support and coordinate TB control between all healthcare levels [2]. One key responsibility of the coordinator is to establish a treatment plan for every individual starting TB treatment. The plan clearly states the responsibilities of all partners involved and seeks to maximize patient-involvement.

The objective of this study is to measure LTBI treatment completion and determinants of non-completion in all individuals notified to MSIS in 2016. We also assess the risk of severe adverse events related to treatment, and their consequences for the LTBI treatment strategy, and explore the use of associated healthcare resources.

\section{Methods}

\section{Study participants}

This is a nationwide prospective cohort study, including all individuals reported with LTBI treatment to MSIS between January 1, 2016 and December 31, 2016. The protocol is available as supplementary material (Additional file 1:). Data included demographic and clinical information available through MSIS, and additional data on treatment completion, adverse events, patient support, and use of healthcare resources collected through a standardized treatment completion form that was sent to prescribing clinicians and TB coordinators at the time the individual was reported to MSIS (form with translation, see Additional file 2: $a, b$ ). If the form was not returned, we sent one reminder by mail before calling the prescribing clinician or responsible TB coordinator. If multiple forms were returned for the same individual, we verified conflicting data with a call to the responsible physician or TB coordinator. If we were unable to verify conflicting data, such as the number of visits with a physician or TB coordinator, we calculated the mean value of the reported data before inclusion in the analyses. 


\section{Definitions}

LTBI treatment completion was measured as reported by the responsible clinician, and verified by the duration of treatment (measured in days) for the different regimens. Information on adverse events was only reported if it led to interruption (treatment was stopped temporarily, but later continued) or termination (stopped completely) of treatment. Severity of hepatotoxicity was based on reported increase in the liver enzymes alanine aminotransferase (ALAT) and aspartate transaminase (ASAT) and bilirubin in blood samples. Severity was classified by upper limits of normal values (ULN) for serum levels of ALAT or ASAT, consistent with the Common Terminology Criteria for Adverse Events (CTCAE4) [17]: grade 1 ( $>\mathrm{ULN}-3.0 \mathrm{x} \mathrm{ULN})$, grade 2 $(>3.0-5.0 \times \mathrm{ULN})$, grade $3(>5.0-20.0 \times \mathrm{ULN})$, and grade 4 (> $20.0 \times \mathrm{ULN})$. Other blood test results were not routinely collected. Remaining adverse events were classified by their clinical symptoms.

\section{Statistical analysis}

We used STATA 2015 for statistical analysis. We present descriptive statistics for continuous variables as means and standard deviations for symmetrical data, and medians and interquartile range (IQR) for skewed data. Pearson's chi squared or Fisher's exact test, as appropriate, assessed associations between pairs of variables.

Additionally, we ran two separate logistic regression models. The first model analysed the association between treatment non-completion and origin (Norwegian-born/foreign-born), age $(\geq \mid<35$ years), gender, and type of treatment support (self-administered treatment/ daily DOT/weekly DOT). Treatment regimen could not be included in the model because of its association with DOT. Similarly, we could not include both establishment of treatment plan and use of DOT in the model because the two are related. The final model did not include either the establishment of a treatment plan or immunosuppression because these were not found to be significantly associated with treatment completion in crude analyses.

To investigate if the association between treatment non-completion and treatment support was modified by age, sex, or origin we ran separate models. We ran the baseline model (treatment non-completion $=$ treatment support + origin + age + sex) and then subsequently tested for interaction between treatment support and age, sex, or origin, i.e. (treatment support*age)/ (treatment support"sex)/ (treatment support*origin). We then performed likelihood ratio tests comparing the separate models to the baseline model. We found no statistically significant effect of age or sex on treatment support. We found a significant effect of origin on treatment support and therefore stratified the effect of treatment support on treatment non-completion by origin in the model. We also tested for an interaction between origin and age and origin and sex. No such interactions were identified.

Information on age and origin was complete. Information on treatment completion was missing for six individuals, and treatment support was missing for 13. Since missing information is unlikely random, we used multiple imputations to investigate the effect of missing data on the model. We imputed five datasets using chained equations ("mi impute chained" command) and recreated the complete-case analyses using the imputed data.

The second model analysed the association between interruption or termination of treatment because of adverse events (yes/no), and treatment regimens (3HR/3RPH/Other), age $(>/<35$ years), and immunosuppression (yes/no). One individual had missing information for treatment regimen, and for this person we coded treatment regimen as "other".

To investigate if the association between adverse events and treatment regimen was modified by age or immunosuppression, we ran two models. We ran the baseline model (adverse effect $=$ treatment regimens + age $+\mathrm{im}$ munosuppression) and subsequently tested for interaction between treatment regimens and age or immunosuppression, i.e. (treatment regimens"age)/ (treatment regimen$\mathrm{s}^{*}$ immunosuppression). We also ran a model investigating whether there was an interaction between age and immunosuppression, i.e. (age ${ }^{*}$ immunosuppression). We then performed likelihood ratio tests comparing the separate models to the baseline model. We found no statistically significant effect of age or immunosuppression on treatment regimens. We could not test the interaction between age and immunosuppression due to the small number of cases.

\section{Results}

\section{Study population}

In 2016, MSIS received 747 notifications of the prescription of LTBI treatment. We later excluded 21 cases because the treatment was completed in 2015 (delay in reporting), which left 726 individuals for follow-up in the prospective cohort. Table 1 presents baseline characteristics of the study population by origin.

Eighteen percent of the individuals $(n=131)$ were Norwegian-born and $82 \%(n=595)$ were foreign-born. Information on country of birth was missing for four individuals. Among foreign-born, 197 (33\%) were born in a country with a WHO estimated [18] TB incidence rate (IR) of $\geq 200$ per 100,000 population, Table 1 . Forty-three percent of immigrants $(n=253)$ had no additional risk factors for TB progression other than being foreignborn. Of these, 94 (35\%) arrived from countries with TB IR < 150, 66 (52\%) with IR 150-200, and 91 (46\%) with IR $>200$ per 100,000 population (2 
Table 1 Baseline characteristics of the study population by origin, $n=726$

\begin{tabular}{lllll}
\hline Baseline Characteristics & $\begin{array}{l}\text { Norwegian- } \\
\text { born }\end{array}$ & $\begin{array}{l}\text { Foreign- } \\
\text { born }\end{array}$ & Total & p-value \\
\hline Number of individuals & $131(100)$ & $\begin{array}{l}595 \\
(100)\end{array}$ & $\begin{array}{l}726 \\
(100)\end{array}$ & \\
Gender & & & & 0.115
\end{tabular}

Male

69 (53)

$358(60)$

$427(59)$

Age at notification

$<5$ years
$5-14$ years
$15-34$ years
$35-64$ years
$\geq 65$ years

$37(28)$

25

$10(8)$

$62(9)$

$132(22) \quad 142(20)$

$33(25)$

$315(53) \quad 348(48)$

$\geq 65$ years

$36(27)$

$115(19) \quad 151(21)$

Risk groups $^{\mathrm{a}}$

\begin{tabular}{|c|c|c|c|c|}
\hline Any risk factor ${ }^{a}$ & $121(92)$ & $342(58)$ & $463(64)$ & \\
\hline Recent exposure, contacts & $68(52)$ & $95(16)$ & $163(22)$ & $>0.001$ \\
\hline Contacts $<5$ years & $35(27)$ & $3(0.5)$ & $38(5)$ & \\
\hline Positive chest X-ray, any & $7(5)$ & $60(10)$ & $67(9)$ & 0.090 \\
\hline Fibrotic lesions & $2(2)$ & $17(3)$ & $19(3)$ & 0.388 \\
\hline $\begin{array}{l}\text { Immunosuppressive } \\
\text { condition }\end{array}$ & $51(39)$ & $72(12)$ & $123(17)$ & $>0.001$ \\
\hline HIV infection & - & $12(2)$ & $12(2)$ & \\
\hline Chronic renal disease & $1(1)$ & $4(1)$ & $5(1)$ & \\
\hline Diabetes, any & $1(1)$ & $4(1)$ & $5(1)$ & \\
\hline $\begin{array}{l}\text { Underlying disease relevant } \\
\text { for Immunosuppressive } \\
\text { treatment }{ }^{b}\end{array}$ & $13(10)$ & $10(2)$ & $23(3)$ & \\
\hline Other medical conditions ${ }^{c}$ & $36(7)$ & $42(7)$ & $78(15)$ & \\
\hline $\begin{array}{l}\text { Interferon Gamma Release } \\
\text { Assay (IGRA) }\end{array}$ & & & & $>0.001$ \\
\hline Positive & $94(72)$ & $577(97)$ & $671(92)$ & \\
\hline Negative & $15(11)$ & $5(1)$ & $20(3)$ & \\
\hline Inconclusive & $2(2)$ & - & $2(0)$ & \\
\hline Missing or unknown & $20(15)$ & $13(2)$ & $33(5)$ & \\
\hline Regimen & & & & $>0.001$ \\
\hline 3RH daily' & $97(74)$ & $302(51)$ & $399(55)$ & \\
\hline 3RPH weekly" & $30(23)$ & $276(46)$ & $306(42)$ & \\
\hline $\begin{array}{l}\text { 4R or } 6 \mathrm{H} \text { monotherapy } \\
\text { daily'I }\end{array}$ & $3(2)$ & $15(3)$ & $18(2)$ & \\
\hline Other ${ }^{\text {IV }}$ & - & $2(0)$ & $2(0)$ & \\
\hline Missing & $1(1)$ & - & $1(0)$ & \\
\hline \multicolumn{5}{|c|}{ TB IR (per 100,000) in country of birth" } \\
\hline$<150$ & na & $267(45)$ & $267(45)$ & \\
\hline $150-200$ & na & $127(21)$ & $127(21)$ & \\
\hline
\end{tabular}

Table 1 Baseline characteristics of the study population by origin, $n=726$ (Continued)

\begin{tabular}{llll}
\hline Baseline Characteristics & $\begin{array}{l}\text { Norwegian- } \\
\text { born }\end{array}$ & $\begin{array}{l}\text { Foreign- } \\
\text { born }\end{array}$ & Total \\
\hline$>200$ & na & $197(33)$ & $197(33)$ \\
Missing & na & $4(1)$ & $4(1)$ \\
\hline
\end{tabular}

Data are presented as $\mathrm{n}$ (\%) or median [interquartile range]. \% refers to columns and not rows

${ }^{a}$ According to Norwegian guidelines for the management and control of tuberculosis: with strong or conditional recommendation for LTBI treatment (age $<15$ years, known exposure, positive chest $\mathrm{X}$-ray or immunosuppressive condition)

bIncludes rheumatologic-, dermatologic-, neurologic- and gastroenterological medical conditions

'Other medical conditions include unspecified immunosuppressive conditions reported by the clinician

I $3 \mathrm{RH}$ : rifampicin $(\mathrm{R})$ and isoniazid $(\mathrm{H})$ daily for three months

"3RPH: rifapentine (RP) and isoniazid (H) in 12 weekly doses

IIIRifampicin (R) monotherapy daily for four months or isoniazid $(\mathrm{H})$ monotherapy daily for six months

IV Others include full-course TB treatments for two individuals for two and four months respectively

\# Four immigrants had missing information on country of birth

missing). In total, 314 (53\%) of the immigrants had lived in Norway for less than one year at the time of notification, and were therefore classified as recent immigrants.

The Norwegian-born group was more likely to be part of contact tracing, have at least one medical risk factor, and have a negative interferon-gamma release assay result (IGRA; QuantiFERON TB-Gold $\left(\mathrm{QFT}^{\circ}\right)$ ) prior to treatment onset, Table 1. Age distribution differed between Norwegian and foreign-born, with a higher proportion of either young or old patients in the Norwegian-born group. $3 \mathrm{RH}(55 \%)$ was the most common regimen, although the use of the newest regimen, $3 R P H(42 \%)$, was frequent, and its use increased over the study period. The $3 \mathrm{RPH}$ regimen was more commonly prescribed among foreign-born (46\%) compared to Norwegian-born (23\%).

\section{Treatment completion}

Information on treatment completion was obtained for 719 (99\%) of the individuals who started LTBI treatment. Table 2 presents treatment completion and the reasons for not completing treatment. Overall, 91\% completed their treatment course as reported by the prescribing physician.

Treatment completion did not differ significantly for different regimens $(p=0.124)$. Foreign-born individuals were more likely to complete treatment compared to Norwegian-born [foreign-born, $n=562 \quad(92 \%)$ vs Norwegian-born, $n=115$ (85\%), $p=0.007$ ]. They were also more likely to be prescribed with $3 \mathrm{RPH}$ [foreign-born, $n=276(46 \%)$ vs Norwegian-born $n=30(23 \%) p$-value 0.001 ], a regimen for which DOT is recommended. Foreign-born individuals were also more likely to be treated under DOT, even for regimens for which DOT is not routinely recommended [foreign-born, $n=151$ (47\%) vs Norwegian-born $n=28$ (28\%) $p=0.002$ ]. Among recent immigrants, 294 (94\%) completed their treatment, $48 \%$ 
Table 2 Treatment completion and reasons for non-completion by treatment regimen, Norway 2016

\begin{tabular}{|c|c|c|c|c|}
\hline Treatment completion & $\begin{array}{l}3 \mathrm{RH}^{\prime} \\
\text { daily }\end{array}$ & $\begin{array}{l}3 \mathrm{RPH}^{\prime \prime} \\
\text { weekly }\end{array}$ & Other III & Total \\
\hline Number of individuals & 399 & 306 & 21 & 726 \\
\hline Duration of treatment (days), median [IQR] & 90 [73-156] & $77[70-98]$ & - & - \\
\hline \multicolumn{5}{|l|}{ Treatment completion } \\
\hline Completed according to physician ${ }^{a}$ & $357(89.5)$ & $284(92.8)$ & $17(80.9)$ & $658(90.6)$ \\
\hline Missing information & $3(0.8)$ & $4(1.3)$ & - & $7(1.0)$ \\
\hline Incomplete treatment & $39(10)$ & $18(5.9)$ & $4(19.0)$ & $61(8.4)$ \\
\hline \multicolumn{5}{|l|}{ Reasons for incomplete treatment } \\
\hline LTBI excluded & $4(1.0)$ & - & - & $4(0.6)$ \\
\hline Diagnosed with TB disease & $1(0.3)$ & - & & $1(0.1)$ \\
\hline Patient choice & $4(1.0)$ & $2(0.7)$ & - & $6(0.8)$ \\
\hline Lost to follow-up & $1(0.3)$ & $3(1.0)$ & - & $4(0.6)$ \\
\hline Other or unknown & $3(0.8)$ & $2(0.7)$ & & $5(0.7)$ \\
\hline Termination due to adverse effects $^{b}$ & $26(6.5)$ & $11(3.4)$ & $4(19.0)$ & $41(5.6)$ \\
\hline Hepatotoxicity (grade $1-2)^{c}$ & $8(2.0)$ & - & - & $8(1.1)$ \\
\hline Hepatotoxicity (grade $3-4)^{c}$ & $5(1.6)$ & $2(0.7)$ & $1(4.8)$ & $8(1.1)$ \\
\hline Gastrointestinal symptoms & $10(2.5)$ & $8(2.6)$ & $2(9.5)$ & $20(2.8)$ \\
\hline Fatigue & $6(1.5)$ & $8(2.6)$ & $1(4.8)$ & $15(2.1)$ \\
\hline Flu-like symptoms & $2(0.5)$ & $4(1.3)$ & $1(4.8)$ & $7(1.0)$ \\
\hline Skin rash & $2(0.5)$ & $1(0.3)$ & $1(4.8)$ & $4(0.6)$ \\
\hline Peripheral neuropathy & $1(0.3)$ & & & $1(0.1)$ \\
\hline Joint pain & - & $2(0.6)$ & - & $2(0.3)$ \\
\hline Other symptoms ${ }^{d}$ & $2(0.5)$ & $3(1.0)$ & $2(9.5)$ & $7(1.0)$ \\
\hline
\end{tabular}

Data are presented as $\mathrm{n}(\%)$ or median [interquartile range]

' $3 \mathrm{RH}$ : rifampicin $(\mathrm{R})$ and isoniazid $(\mathrm{H})$ daily for three months

"3RPH: rifapentine (RP) and isoniazid (H) in 12 weekly doses

IIIOther: rifampicin (R) monotherapy daily for four months $(n=5)$, Isoniazid (H) monotherapy for six months $(n=13)$ or combination therapy for TB disease ( $n=2)$ and missing information about drug regimen $(n=1)$

*Duration of treatment for those where the clinician reported the treatment as completed

${ }^{a}$ The responsible clinician reported that the planned treatment was completed

${ }^{b}$ Many reported more than one adverse effect

'Severity of hepatotoxicity was classified according to Common Terminology Criteria for Adverse Events (CTCAE), ULN $=$ upper limits of normal value for serum levels of liver function, grade 1 (>ULN $-3.0 \times$ ULN), grade $2(>3.0-5.0 \times$ ULN), grade $3(>5.0-20.0 \times$ ULN), and grade 4 (> 20.0 $\times$ ULN)

${ }^{\mathrm{d}}$ Includes headache, sleep disorder, and unstable international normalized ratio (INR) for prothrombin time

were prescribed with $3 \mathrm{RPH}$, and $65 \%$ were treated under DOT while on regimens other than $3 \mathrm{RPH}$.

In crude logistic regression models, origin, age, sex, and treatment support were significantly associated with treatment non-completion. In a multivariable logistic regression model, we found that neither origin, age over 35 years, or sex were significantly associated with treatment non-completion. However, the effect of treatment support was modified by origin, with significantly lower risk of non-completion with daily and weekly DOT compared to self-administration in the foreign-born group. Treatment support had no significant effect on treatment completion in the Norwegian-born group, Table 3.

We ran a sensitivity analysis by rerunning the analyses in Table 3 with a multiple imputed dataset (using chained equations). The sensitivity analysis showed no impact on the overall results, and the results are available as supplementary material (Additional file 3).

\section{Adverse events}

Adverse events was the most common reason reported for incomplete treatment in all groups. The total number of adverse events according to the study definitions was 47 (6.5\%). In total, 41 (5.6\%) individuals terminated treatment, Table 2, and an additional six (0.8\%) individuals interrupted treatment to control adverse events but later continued and completed treatment. These six individuals are recorded as complete treatment in Table 2 . Three of these temporary interruptions $(3 \mathrm{RH})$ were due to hepatotoxicity (one grade 2 and two grade 3), two were due to gastrointestinal symptoms, (3RPH and $\mathrm{H})$ 
Table 3 Associations between the outcome of treatment noncompletion and the variables treatment support, origin, age and $\operatorname{sex}(n=726)$

\begin{tabular}{|c|c|c|c|c|c|c|c|}
\hline \multirow[t]{2}{*}{ Covariates } & \multicolumn{3}{|c|}{ Univariable } & \multicolumn{4}{|c|}{ Multivariable } \\
\hline & $n$ & $\mathrm{COR}$ & $\mathrm{p}$ & $a \mathrm{OR}$ & SE & $p$ & $95 \% \mathrm{Cl}$ \\
\hline \multicolumn{8}{|l|}{ Origin } \\
\hline Foreign-born & 595 & 1 (ref) & & 1 (ref) & & & \\
\hline Norwegian-born & 131 & 2.1 & 0.016 & 0.8 & 0.342 & 0.602 & $0.34-1.84$ \\
\hline \multicolumn{8}{|l|}{ Age group } \\
\hline$\leq 35$ & 561 & 1 (ref) & & 1 (ref) & & & \\
\hline$>35$ years & 165 & 1.9 & 0.026 & 1.7 & 0.521 & 0.093 & $0.92-3.09$ \\
\hline \multicolumn{8}{|l|}{ Sex } \\
\hline Female & 299 & 1 (ref) & & 1 (ref) & & & \\
\hline Male & 427 & 0.5 & 0.017 & 0.6 & 0.093 & 0.106 & $0.36-1.10$ \\
\hline
\end{tabular}

Treatment support. Model with interaction term for origin: LRT $p$-value of interaction term $=0.049$

Effect of treatment support on treatment completion in foreign-born (multivariate wald p-value 0.017)

\begin{tabular}{lllllllll} 
Self-administered $^{\mathrm{a}}$ & 174 & 1 (ref) & \multicolumn{5}{c}{1 (ref) } \\
DOT daily $^{\mathrm{b}}$ & 151 & 0.2 & 0.005 & 0.3 & 0.150 & 0.017 & $0.10-0.80$ \\
DOT weekly $^{\mathrm{c}}$ & 261 & 0.4 & 0.009 & 0.4 & 0.162 & 0.027 & $0.22-0.91$
\end{tabular}

Effect of treatment support on treatment completion in Norwegian-born (multivariate wald p-value 0.468)

\begin{tabular}{llllllll} 
Self-administered $^{\mathrm{a}}$ & 80 & 1 (ref) & \multicolumn{1}{c}{1 (ref) } \\
DOT daily $^{\mathrm{b}}$ & 28 & 1.7 & 0.374 & 2.1 & 1.332 & 0.229 & $0.62-7.26$ \\
DOT weekly & 19 & 0.9 & 0.928 & 1.0 & 0.868 & 0.961 & $0.20-5.34$ \\
\hline
\end{tabular}

OR Odds ratio, SE standard error, DOT Direct Observed Treatment for part of or the full treatment period

${ }^{\text {a}}$ Self-administered include those who managed their treatment themselves or were given weekly pill boxes

bdaily DOT: those who were administered daily treatment under direct observation 'weekly DOT: those who were administered weekly rifapentine and isoniazid under direct observation and one to fatigue (3RPH). The duration of the interruption was 2, 4, 7, 22, 28 and 113 days respectively.

In a multivariable logistic regression model, we found that regimen was borderline associated with adverse events $(p$-value $=0.065)$. There were no significant differences when comparing $3 \mathrm{RH}$ vs $3 \mathrm{RPH}$, nor $3 \mathrm{RH}$ vs Other ( $\mathrm{R}$ or $\mathrm{H}$ monotherapy or full course $\mathrm{TB}$ treatment). However, there were significantly fewer adverse events with 3RPH compared to "other" regimens ( $p=0.037)$. Age over 35 years was significantly associated with having more adverse events, even after adjusting for regimen, whereas immunosuppression was not significantly associated with having adverse events after adjusting for other variables, Table 4 . No deaths were reported.

Duration of treatment prior to termination ranged from 2 to 122 days, with a median of 25 days (IQR 6-84). For the two 3-month regimens combined (3RH $+3 R P H), 61 \%$ of those who terminated treatment completed less than half of the prescribed regimen.

Among those who met the study-definition for adverse effects, 22 (47\%) had one symptom, 16 (34\%) had two, and nine (19\%) had three. There was no significant difference in number of symptoms reported between those who interrupted and those who terminated treatment ( $p$ =0677). Gastrointestinal symptoms was the most common complaint in both age groups, followed by hepatotoxicity and fatigue, Table 2. Two individuals were classified with grade 4 hepatotoxicity. One was a child who was diagnosed with toxic hepatitis after two weeks of LTBI treatment. Treatment was immediately stopped, allowing transaminase values to normalize, and the patient fully recovered. The other was a middle-aged individual who developed hepatotoxicity with high transaminase values after eight weeks of LTBI treatment.

Table 4 Associations between adverse events ${ }^{a}$, regimen, age, and immunosuppression, $n=726$

\begin{tabular}{|c|c|c|c|c|c|c|c|}
\hline \multirow[t]{2}{*}{ Covariates } & \multirow[b]{2}{*}{$\mathrm{n}$} & \multicolumn{2}{|c|}{ Univariable } & \multicolumn{4}{|c|}{ Multivariable } \\
\hline & & $\mathrm{cOR}$ & $\mathrm{p}$ & $\mathrm{a} O R$ & SE & $\mathrm{p}$ & $95 \% \mathrm{Cl}$ \\
\hline \multicolumn{8}{|c|}{ Regimen (Wald test for overall variable in adjustment model, $p=0.065$ ) } \\
\hline $3 \mathrm{RH} \mathrm{H}^{\mathrm{b}}$ & 399 & 1 (ref) & & 1 (ref) & & & \\
\hline $3 \mathrm{RPH}^{\mathrm{c}}$ & 306 & 0.57 & 0.097 & 0.60 & 0.209 & 0.141 & $0.30-1.18$ \\
\hline Other $^{d}$ & 21 & 3.99 & 0.012 & 2.37 & 1.360 & 0.131 & $0.78-7.30$ \\
\hline \multicolumn{8}{|l|}{ Age } \\
\hline$\leq 35 \mathrm{yrs}$ & 561 & 1 (ref) & & 1 (ref) & & & \\
\hline$>35$ yrs & 165 & 3.62 & $<0.001$ & 2.51 & 2.25 & 0.024 & $1.13-5.60$ \\
\hline \multicolumn{8}{|l|}{ Immunosuppression } \\
\hline No immunosuppression & 603 & 1 (ref) & & 1 (ref) & & & \\
\hline Immunosuppression, any & 123 & 3.39 & $<0.001$ & 1.55 & 0.66 & 0.306 & $0.67-3.57$ \\
\hline
\end{tabular}

OR Odds ratio, SE standard error

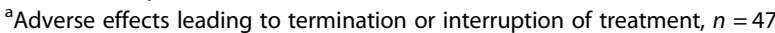

$\mathrm{b}_{3 \mathrm{HR}, 3}$ months daily rifampicin and isoniazid

' $3 \mathrm{RPH}, 12$ weekly doses of rifapentine and isoniazid

${ }^{\mathrm{d} O t h e r ; ~ r i f a m p i c i n ~ m o n o t h e r a p y ~}(n=5)$, isoniazid monotherapy $(n=13)$, combination therapy for TB disease $(n=2)$ and 1 missing information 
The treatment was immediately stopped, and transaminase values normalized after six weeks. Both individuals received $3 \mathrm{RH}$ regimen and were managed as outpatients. Additionally, eight individuals were classified as having hepatotoxicity grade 3 . Two of these were hospitalized due to complex comorbidities. The remaining were treated as outpatients, and transaminase values normalized after treatment was stopped. The $3 \mathrm{RH}$ regimen was prescribed in 16 out of 19 (84\%) patients who interrupted or terminated treatment due to hepatotoxicity.

\section{Patient support, drug administration, and use of healthcare resources}

A treatment plan was established prior to treatment for $655(90 \%)$ individuals. Foreign-born individuals were more likely to have a treatment plan compared with Norwegian-born individuals $(n=544,91 \%$ vs $n=111$, $85 \%$, p 0.017).

DOT, either for part of or the whole treatment period, was reported in 173 (43\%) of individuals prescribed with $3 \mathrm{RH}$, in $280(92 \%)$ with $3 \mathrm{RPH}$, and in six (29\%) with "other", Table 5. Home-visiting nurses provided DOT in 324 (71\%) cases, the family in 11 (2\%), and outpatient hospital clinics, assisted living facilities, refugee centres, work places, or general practitioners in 57 (12\%). Information about the DOT provider was missing for 65 (15\%) individuals.

The median number of consultations with a medical doctor was 1.5 (IQR 1-8, range 1-11), and 1.5 (IQR 0-7, range $0-14)$ with nurse. Almost half (48\%) had only one consultation with a doctor. We found no significant difference in the mean number of consultations with a doctor or nurse between foreign-born and Norwegian-born individuals. Among the 658 individuals who completed treatment, 397 (60\%) had their last consultation in the hospital before $(n=334,51 \%)$, or at the time of treatment initiation $(n=63,9 \%)$. Only $193(29 \%)$ had a consultation in the hospital more than six weeks after the treatment start date.

Table 5 Drug-administration by regimen, $\mathrm{n}=726$

\begin{tabular}{|c|c|c|c|c|}
\hline Drug administration & $3 R H^{a}$ daily & $3 \mathrm{RPH}^{\mathrm{b}}$ weekly & Other $^{\mathrm{c}}$ & Total \\
\hline Number of individuals & $399(100)$ & $306(100)$ & $21(100)$ & $726(100)$ \\
\hline Self-administered & $220(55)$ & $20(7)$ & $14(67)$ & $254(35)$ \\
\hline \multicolumn{5}{|c|}{ Direct observed treatment } \\
\hline Whole period & $132(33)$ & $269(88)$ & $3(14)$ & $404(56)$ \\
\hline Part of period & $41(10)$ & $11(4)$ & $3(14)$ & $55(8)$ \\
\hline Missing information & $6(2)$ & $6(2)$ & $1(5)$ & $13(2)$ \\
\hline
\end{tabular}

Data are presented as $\mathrm{n}(\%)$

${ }^{a}$ rifampicin $(\mathrm{R})$ and isoniazid $(\mathrm{H})$ daily for three months

${ }^{b}$ rifapentine (RP) and isoniazid (H) in 12 weekly doses

${ }^{c}$ rifampicin $(R)$ monotherapy daily for four months $(n=5)$, Isoniazid $(H)$ monotherapy for six months $(n=13)$ or combination therapy for TB disease $(n=2)$ and missing information $(n=1)$

${ }^{\mathrm{d}}$ Other included: hospital outpatient clinic, assisted living facilities, refugee centres, work-places or with general practitioners.

\section{Discussion}

This one-year prospective study found a 91\% LTBI treatment completion rate in Norway. Treatment completion did not differ significantly by regimen. Completion was higher among foreign-born than Norwegian-born individuals. Treatment under DOT had a significant effect on treatment completion for foreign-born individuals, but not in the Norwegian-born group. The primary reason for not completing treatment was adverse events. The majority of these were mild to moderate in severity, although medically significant (grade 3 ) and severe (grade 4) hepatotoxicity led to termination of treatment in $1.1 \%$ of participants. Few individuals were lost to follow-up. Individual treatment plans were established for the majority, and DOT was common, even with regimens where this is not routinely recommended.

\section{Treatment completion}

The treatment completion rate of $91 \%$ is consistent with the $89 \%$ previously reported in Norway [13]. Direct comparison with previous studies is difficult due to differences in study designs, regimens, and the populations under study. Completion rates are commonly reported as inversely related to treatment length [7]. Unsupervised six to nine months of isoniazid treatments in the US have shown around $50 \%$ completion rates $[10,11$, 19, 20], 3-4 months RH regimens are reported at 85$90 \%[13,21-23]$ and recent studies report similarly high completion rates with weekly (supervised) $3 \mathrm{RPH}$ administration [24-28]. Our results are consistent with a 2017 review [29] reporting no important differences in efficacy and completion rates for the $3 \mathrm{RPH}$ regimen when compared to other regimens. The review, however, did support a higher likelihood of completion with a shorter regimen. Additionally, lower loss to follow up has been associated with immunocompromised patients, being part of a contact investigation, and the shorter, rifamycin-based regimens [30].

Our high completion rate is surprising given that the majority of patients were recent arrivals to Norway and were born in countries with a high TB prevalence, factors often associated with poor access and adherence to health services. A Swedish study reported 76\% LTBI treatment completion over a six year period (2002-2007) overall, but only $68 \%$ completion among recent immigrants, $(<1$ years residence) with immigrants of Somali origin having the lowest completion rates [23]. Similarly in Japan, a study reported significantly higher completion rates in Japanese versus foreign-born individuals [31]. In our setting, the most recent immigrants completed treatment at a higher rate than other immigrants, and immigrants as a whole completed treatment to a higher extent than Norwegian-born individuals. This finding is unexpected because immigrants in Norway tend to under-utilize other 
preventive health services, such as mammography [32]. Foreign-born individuals in this study were more likely to have a treatment plan established prior to LTBI treatment, be treated under direct observation, and were more commonly prescribed the $3 \mathrm{RPH}$ weekly regimen compared to Norwegian-born individuals. These findings may explain such high rates of completion among recent immigrants. Treatment support, which was coded separately for daily or weekly DOT to reflect treatment regimens, was significantly associated with treatment completion among foreign-born individuals when controlling for other co-variates. However, we were unable to control for adverse effects as a co-variate in the analyses, since this information was only recorded when not completing treatment.

Another explanation may be the role of TB coordinators, mostly specialized nurses, which is unique in a northern European setting. The TB coordinators are actively involved in implementation of TB control activities, both on individual and system levels. They know their patients well, and this may mitigate loss to follow-up. Previous studies have shown that social interventions such as adherence coaching, contingency contracting, enhanced outreach, and home visits improve treatment completion and may benefit patients who are at risk of progressing to active TB [33, 34]. In Norway, such activities are initiated by TB coordinators.

\section{Adverse events}

Adverse events was the most common reason for not completing treatment in both the Norwegian and foreign-born groups. This is consistent with other studies [35]. The proportion of individuals who discontinued LTBI treatment due to side-effects (5.6\%) in this study is similar to the $7.6 \%$ reported in the Netherlands [21], 6.4 and $5.9 \%$ reported in the USA [35], and 7\% reported in Norway in 2009 [13], but lower than the 12\% reported from Australia [36]. Similarly, a recent study in Taiwan that compared treatment completion and cost effectiveness for $3 \mathrm{RPH}$ versus $\mathrm{H}$ reported higher completion in the $3 \mathrm{RPH}$ regimen due to the shorter treatment period and reduced likelihood of severe side effects [37]. This is also consistent with our results.

The severity of reported adverse events varied. Since the treatment is preventive rather than curative, mild to moderate side effects may influence both the patient's and the doctor's motivation to continue treatment. The rate of clinically significant and severe hepatotoxicity (grade 3 and 4) reported in this study (1.1\%) is lower than a median rate of $1.8 \%$ (range $0.1-11.9 \%$ ) reported in a study on age-related hepatotoxicity following 6-9 months of isoniazid [16]. In accordance with other studies, we found a higher rate of grade 3 and 4 hepatotoxicity with increasing age $[7,16,36]$, lower rates with $3 \mathrm{RPH}$ compared with other regimens $[20,26,35,38]$, rare need for hospitalization, and no deaths [13, 16, 27, 36, 39, 40].

\section{Patient support and use of health care resources}

Norway's high completion rate must also be seen in relation to the resources used to achieve it. Two elements that make preventive treatment in Norway relatively costlier than elsewhere are the frequent use of DOT and the highly specialized care levels. Almost two thirds of the patients involved in the study received treatment under DOT. Norwegian guidelines do not routinely recommend DOT for treatment regimens other than $3 \mathrm{RPH}$. The increasing use of this 12-dose regimen (3RPH), with weekly administration rather than a prolonged daily regimen, will reduce DOT associated costs. Further, a recent randomized clinical trial found that there was not a significant difference in completion rates for directly observed $3 R P H$ versus self-administered $3 R P H$ treatment, which may change current recommendations for supervised $3 \mathrm{RPH}$ treatment [41].

Providing preventive treatment through subspecialist physicians rather than primary care is costly. However, training a larger group of primary care doctors about the details of $\mathrm{TB}$ and its prevention and treatment in low incidence settings such as Norway would require substantial effort. The greatest cost, as well as the greatest benefit, may be attributable to having dedicated TB coordinators. In the current study, $90 \%$ of patients had treatment plans established by TB coordinators.

\section{Strengths and limitations}

The strengths of the study include the population-wide approach, the comprehensive information available for every individual, the standardized classification of severity of hepatotoxicity, and our very low rate of loss to follow-up. The sensitivity of our reporting system for LTBI treatment is assumed to be high since information on TB-related prescriptions is collected independently, and missing data is routinely monitored to ensure complete information.

Limitations include that completion rates and adverse events for the various regimens may reflect systematic differences in treatment assignment and clinician assessment rather than differences in the regimens themselves. We have, when possible, adjusted for this in our analyses. Judgment of treatment completion was made by the treating physician, often based on only one consultation at the beginning of the treatment course, in addition to information received from the $\mathrm{TB}$ coordinator and the DOT-provider. The question about completion was, however, followed up with a statement of the number of days on treatment and overseen by the TB coordinator. Adverse drug events that did not lead to interruption or 
termination of treatment were not recorded. Therefore, we could not control for this as a co-variate. We may have also missed some adverse effects and mild degrees of toxicity.

\section{Conclusions}

This study indicates that with well-organized health services, high LTBI treatment completion is possible, even in high-risk immigrant groups. We report high treatment completion across all subgroups. Treatment completion is the end-point of Norway's LTBI screening strategy. Knowledge of the treatment completion rate and adverse events are crucial in the assessment of the screening program's impact and cost effectiveness. Although adverse events were the primary determinants of treatment non-completion, our results seem reassuring, even for more elderly and immunocompromised individuals, given adequate monitoring and follow-up during treatment. While this study has shown that DOT is very effective in ensuring treatment completion among hard to reach populations, further research is necessary to evaluate best practices from a cost-effectiveness standpoint.

\section{Additional files}

\section{Additional file 1: protocol. (PDF $376 \mathrm{~kb}$ )}

Additional file 2: a, form sent to clinicians for information about treatment completion. b, translation of form sent to clinicians for information about treatment completion. (ZIP $680 \mathrm{~kb}$ )

Additional file 3: a sensitivity analysis exploring the associations between treatment non-completion, and treatment support, origin, age and sex after imputation for missing values. (PDF $472 \mathrm{~kb}$ )

\section{Abbreviations}

3RH: Daily Rifampicin and Isoniazid for 3 Months; 3RPH: Weekly Rifapentine and Isoniazid for 3 months; ALAT: Alanine aminotransferase; ASAT: Aspartate aminotransferase; CTCAE: Common Terminology Criteria for Adverse Events; DOT: Directly Observed Treatment; H: Daily Isoniazid for 6 months; IGRA: Interferon-gamma release assay; IR: Incidence Rate; LTBI: Latent Tuberculosis Infection; MSIS: Norwegian Surveillance System for Infectious Diseases; NIPH: Norwegian Institute of Public Health; R: Daily Rifampicin for 4 months; TB: Tuberculosis; ULN: Upper Limits of Normal values; WHO: World Health Organization

\section{Acknowledgements}

We thank clinicians and tuberculosis coordinators throughout Norway for their strong efforts in submitting treatment completion forms for the study. We also thank Kirsten Konsmo and Annette Tyvand at the Norwegian Institute of Public Health for their support in data-collection and data-cleaning. Finally, we thank the U.S.-Norway Fulbright Foundation for their support of YS's efforts on this project.

\section{Funding}

The Norwegian Health association funded the project leader (BAW) and a Fulbright Grant funded the first author (YS) throughout the study period. The Norwegian Institute of Public Health allocated administrative resources to the project.

\section{Availability of data and materials}

The datasets generated and/or analysed during the current study are not publicly available due to regulations in the Norwegian Health Research Act and the Norwegian Data Protection Act for use (and storage) of Personal
Data related to health, but are available from the corresponding author on reasonable request.

\section{Authors' contributions}

BAW initiated the study, wrote the protocol, obtained necessary approvals and set up the data-collection. All authors were involved in the final version of the protocol. YS was responsible for the major part of data-collection and data-entry, data-analyses, and for drafting the manuscript. TM evaluated and classified adverse drug events, RW was responsible for the statistical analyses, BAW and YS drafted the manuscript and YS, HEA, TMA, AMDR, HT, RW and BAW critically revised and approved the final version of the manuscript.

\section{Ethics approval and consent to participate}

The study was approved by the Regional Committees for Medical and Health Research Ethics (REC South East, ref. number 2015/2122). As part of the approval exemption from consent to participate was waived based on The Health Research Act \$35, §9 and §33; (i) notification of LTBI treatment in Norway is mandatory without asking for consent, (ii) treatment initiation not affected by the study, (iii) screening for TB is mandatory, which makes the monitoring and evaluation of the yield and safety of screening essential.

\section{Consent for publication}

Not applicable.

\section{Competing interests}

The authors declare that they have no competing interests.

\section{Publisher's Note}

Springer Nature remains neutral with regard to jurisdictional claims in published maps and institutional affiliations.

\section{Author details}

${ }^{1}$ Perelman School of Medicine at the University of Pennsylvania, Philadelphia, PA, USA. ²Department of Pulmonary Medicine, Stavanger University Hospital, Stavanger, Norway. ${ }^{3}$ Department of Pulmonary Medicine, TB unit, Stavanger University Hospital, Stavanger, Norway. ${ }^{4}$ Department of Tuberculosis, Blood Borne and Sexually Transmitted Infections, Norwegian Institute of Public Health, Oslo, Norway. ${ }^{5}$ Department of Infectious Diseases, Oslo University Hospital, Oslo, Norway. ${ }^{6}$ Institute of Clinical Medicine, University of Oslo, Oslo, Norway. ${ }^{7}$ Dep. of Clinical Science, University of Bergen, Oslo, Norway. ${ }^{8}$ Department of Pulmonary Medicine, Oslo University Hospital, Oslo, Norway. ${ }^{9}$ Department of Infectious Disease Epidemiology and Modelling, Norwegian Institute of Public Health, Oslo, Norway. ${ }^{10}$ Department of Vaccine Preventable Diseases, Norwegian Institute of Public Health, Oslo, Norway.

Received: 25 January 2018 Accepted: 31 October 2018

Published online: 19 November 2018

References

1. WHO. Global strategy and targets for tuberculosis prevention, care and control after 2015 [http://apps.who.int/gb/ebwha/pdf_files/EB134/B134_12en.pdf?ua $=1]$.

2. Helse- og omsorgsdepartementet: Forskrift om tuberkulosekontroll FOR2009-02-13-205; 2009.

3. Norwegian Institute of Public Health Guidelines for Prev Control of tuberculosis [https://www.fhi.no/nettpub/tuberkuloseveilederen/].

4. Arnesen TM, Heldal E, Mengshoel AT, Nordstrand K, Rønning K. In: Folkehelseinstituttet, editor. Tuberkulose i Norge i 2016 med behandlingsresultater for 2015. Oslo: Norwegian Institute of Public Health 2017. isbn:ISSN 1894-4868.

5. Arnesen TM, Eide KA, Norheim G, Mengshoel AT, Sandbu S, Winje BA. In: Folkehelseinstituttet, editor. Tuberkulose i Norge i 2013 med behandlingsresultater for 2012; 2014

6. Helse- og omsorgsdepartementet: Forskrift om Meldingssystem for smittsomme sykdommer (MSIS-forskriften) FOR-2003-06-20-740. 2003.

7. WHO. Guidelines for the management of latent tuberculosis infection [http://www.who.int/tb/publications/ltbi_document_page/en/].

8. Sharma SK, Sharma A, Kadhiravan T, Tharyan P. Rifamycins (rifampicin, rifabutin and rifapentine) compared to isoniazid for preventing tuberculosis in HIV-negative people at risk of active TB. Cochrane Database Syst Rev. 2013;7:CD007545 
9. Aspler A, Long R, Trajman A, Dion MJ, Khan K, Schwartzman K, Menzies D. Impact of treatment completion, intolerance and adverse events on health system costs in a randomised trial of 4 months rifampin or 9 months isoniazid for latent TB. Thorax. 2010;65(7):582-7.

10. Horsburgh CR Jr, Goldberg S, Bethel J, Chen S, Colson PW, HirschMoverman Y, Hughes S, Shrestha-Kuwahara R, Sterling TR, Wall K, et al. Latent TB infection treatment acceptance and completion in the United States and Canada. Chest. 2010;137(2):401-9.

11. Page KR, Sifakis F, Montes de Oca R, Cronin WA, Doherty MC, Federline L, Bur S, Walsh T, Karney W, Milman J, et al. Improved adherence and less toxicity with rifampin vs isoniazid for treatment of latent tuberculosis: a retrospective study. Arch Intern Med. 2006;166(17):1863-70.

12. Getahun H, Matteelli A, Abubakar I, Aziz MA, Baddeley A, Barreira D, Den Boon S, Borroto Gutierrez SM, Bruchfeld J, Burhan E, et al. Management of latent Mycobacterium tuberculosis infection: WHO guidelines for low tuberculosis burden countries. Eur Respir J. 2015;46(6):1563-76.

13. Olsen AlM, Andersen HE, Amus J, Djupvik JA, Gran G, Skaug K, Mørkve O. Management of latent tuberculosis infection in Norway in 2009: a descriptive cross-sectional study. Public Health Action. 2013;3(2):166-71.

14. Harstad I, Heldal E, Steinshamn SL, Garasen H, Jacobsen GW. Tuberculosis screening and follow-up of asylum seekers in Norway: a cohort study. BMCPublic Health. 2009;9:141.

15. Harstad I, Heldal E, Steinshamn SL, Garasen H, Winje BA, Jacobsen GW. Screening and treatment of latent tuberculosis in a cohort of asylum seekers in Norway. ScandJ Public Health. 2010;38(3):275-82.

16. Kunst $\mathrm{H}$, Khan KS. Age-related risk of hepatotoxicity in the treatment of latent tuberculosis infection: a systematic review. International Journal of Tuberculosis \& Lung Disease. 2010;14(11):1374-81.

17. Common Terminology Criteria for Adverse Events (CTCAE) Version 4. https://www.eortc.be/services/doc/ctc/CTCAE_4.03_2010-06-14_ QuickReference_5x7.pdf.

18. WHO. Global Tuberculosis Report. Geneva: World Health Organization; 2016.

19. Lardizabal A, Passannante M, Kojakali F, Hayden C, Reichman LB. Enhancement of treatment completion for latent tuberculosis infection with 4 months of rifampin. Chest. 2006;130(6):1712-7.

20. Perez AP, Seo SK, Schneider WJ, Eisenstein C, Brown AE. Management of Latent Tuberculosis Infection among Health Care workers: 10-year experience at a single center. Clin Infect Dis. 2017;65(12):2105-11.

21. Erkens CG, Slump E, Verhagen M, Schimmel H, de Vries G, Cobelens F, van den Hof S. Monitoring latent tuberculosis infection diagnosis and management in the Netherlands. Eur Respir J. 2016;47(5):1492-501.

22. Geijo MP, Herranz CR, Vano D, Garcia AJ, Garcia M, Dimas JF. Short-course isoniazid and rifampin compared with isoniazid for latent tuberculosis infection: a randomized clinical trial. Enferm Infecc Microbiol Clin. 2007;25(5):300-4

23. Kan B, Kalin M, Bruchfeld J. Completing treatment for latent tuberculosis: patient background matters. Int J Tuberc Lung Dis. 2013;17(5):597-602.

24. Lines $G$, Hunter P, Bleything S. Improving treatment completion rates for latent tuberculosis infection: a review of two treatment regimens at a community health center. J Health Care Poor Underserved. 2015;26(4):1428-39.

25. Martinson NA, Barnes GL, Moulton LH, Msandiwa R, Hausler H, Ram M, McIntyre JA, Gray GE, Chaisson RE. New regimens to prevent tuberculosis in adults with HIV infection. N Engl J Med. 2011;365(1):11-20.

26. Sterling TR, Villarino ME, Borisov AS, Shang N, Gordin F, Bliven-Sizemore E, Hackman J, Hamilton CD, Menzies D, Kerrigan A, et al. Three months of rifapentine and isoniazid for latent tuberculosis infection. N Engl J Med. 2011;365(23):2155-66.

27. Sandul AL, Nwana N, Holcombe JM, Lobato MN, Marks S, Webb R, Wang SH, Stewart B, Griffin P, Hunt G, et al. High rate of treatment completion in program settings with 12-dose weekly isoniazid and Rifapentine (3HP) for latent Mycobacterium tuberculosis infection. Clin Infect Dis. 2017. https://doi.org/10.1093/cid/cix505.

28. McClintock AH, Eastment M, McKinney CM, Pitney CL, Narita M, Park DR, Dhanireddy S, Molnar A. Treatment completion for latent tuberculosis infection: a retrospective cohort study comparing 9 months of isoniazid, 4 months of rifampin and 3 months of isoniazid and rifapentine. BMC Infect Dis. 2017;17(1):146

29. Pease C, Hutton B, Yazdi F, Wolfe D, Hamel C, Quach P, Skidmore B, Moher D, Alvarez GG. Efficacy and completion rates of rifapentine and isoniazid (3HP) compared to other treatment regimens for latent tuberculosis infection: a systematic review with network meta-analyses. BMC Infect Dis. 2017;17(1):265
30. Alsdurf H, Hill PC, Matteelli A, Getahun H, Menzies D. The cascade of care in diagnosis and treatment of latent tuberculosis infection: a systematic review and meta-analysis. Lancet Infect Dis. 2016;16(11):1269-78.

31. Kawatsu L, Uchimura K, Ohkado A. Trend and treatment status of latent tuberculosis infection patients in Japan - analysis of Japan TB surveillance data. PLoS One. 2017;12(11):e0186588.

32. Bhargava S, Tsuruda K, Moen K, Bukholm I, Hofvind S. Lower attendance rates in immigrant versus non-immigrant women in the Norwegian breast Cancer screening Programme. J Med Screen. 2017;1:969141317733771.

33. Stuurman AL, Vonk Noordegraaf-Schouten M, van Kessel F, Oordt-Speets AM, Sandgren A, van der Werf MJ. Interventions for improving adherence to treatment for latent tuberculosis infection: a systematic review. BMC Infect Dis. 2016;16:257.

34. Eastment MC, McClintock AH, McKinney CM, Narita M, Molnar A. Factors that influence treatment completion for latent tuberculosis infection. J Am Board Fam Med. 2017;30(4):520-7.

35. Moro RN, Borisov AS, Saukkonen J, Khan A, Sterling TR, Villarino ME, Scott NA, Shang N, Kerrigan A, Goldberg SV. Factors associated with noncompletion of latent tuberculosis infection treatment: experience from the PREVENT TB trial in the United States and Canada. Clin Infect Dis. 2016; 62(11):1390-400.

36. Denholm JT, McBryde ES, Eisen DP, Penington JS, Chen C, Street AC. Adverse effects of isoniazid preventative therapy for latent tuberculosis infection: a prospective cohort study. Drug Healthc Patient Saf. 2014;6:145-9.

37. Huang YW, Yang SF, Yeh YP, Tsao TC, Tsao SM. Impacts of 12-dose regimen for latent tuberculosis infection: treatment completion rate and costeffectiveness in Taiwan. Medicine (Baltimore). 2016;95(34):e4126.

38. Zenner D, Beer N, Harris RJ, Lipman MC, Stagg HR, van der Werf MJ. Treatment of latent tuberculosis infection: an updated network metaanalysis. Ann Intern Med. 2017;167(4):248-55.

39. McElroy PD, ljaz K, Lambert LA, Jereb JA, lademarco MF, Castro KG, Navin TR. National survey to measure rates of liver injury, hospitalization, and death associated with rifampin and pyrazinamide for latent tuberculosis infection. Clin Infect Dis. 2005;41(8):1125-33.

40. Menzies D, Long R, Trajman A, Dion MJ, Yang J, Al Jahdali H, Memish Z, Khan K, Gardam M, Hoeppner V, et al. Adverse events with 4 months of rifampin therapy or 9 months of isoniazid therapy for latent tuberculosis infection: a randomized trial. Ann Intern Med. 2008;149(10):689-97.

41. Belknap R, Holland D, Feng PJ, Millet JP, Cayla JA, Martinson NA, Wright A, Chen MP, Moro RN, Scott NA, et al. Self-administered versus directly observed once-weekly isoniazid and Rifapentine treatment of latent tuberculosis infection: a randomized trial. Ann Intern Med. 2017;167(10):689-97.
Ready to submit your research? Choose BMC and benefit from:
- fast, convenient online submission
- thorough peer review by experienced researchers in your field
- rapid publication on acceptance
- support for research data, including large and complex data types
- gold Open Access which fosters wider collaboration and increased citations
- maximum visibility for your research: over $100 \mathrm{M}$ website views per year
At BMC, research is always in progress.
Learn more biomedcentral.com/submission 